klärbarkeit von politischen Vorgängen signalisiert): „Das Kooperationsverhältnis mit der weißen Oligarchie läßt damit keineswegs etwa westdeutschen Imperialismus diagnostizieren. Vielmehr stellt es sich empirisch als die Verwicklung in die Domination der afrikanischen Konfliktpartei durch die weiße Partei via Kooperation mit letzterer dar" (S. 262).

$\mathrm{Da}$ sich die Studie von Rode auch als Beitrag der nicht herkömmlichen Friedensund Konfliktforschung versteht, sei abschließend noch ein kritisches Wort zu seinem Gewaltbegriff gesagt. Aus all dem was Rode an wertvollen Fakten und Beziehungsmustern aufgearbeitet hat, ergibt bzw. verstärkt sich der Eindruck eines unmenschlichen Rassenregimes in der RSA, das nur durch brutale Aufrechterhaltung von personeller und struktureller Gewalt überleben kann. Die Beziehungsmuster der „Kooperation und Beschwichtigung" seitens der BRD laufen dabei oft ungewollt auf „Systemstabilisierung“ hinaus, wenigstens bisher (S. 273). Angesichts dieser (wie ich meine zutreffenden) Diagnose noch an dem Postulat "gewaltfreier Systemwandel“ festzuhalten, erscheint widersinnig. Im Interesse eines "positiven Friedens" - zu dem sich Rode bekennt - können doch nur Strategien für einen "gewaltabbauenden Systemwandel“ weiterhelfen, der nach aller historischen Erfahrung (Algerien, portugiesische Kolonien, Äthiopien etc.) nicht gewaltfrei verläuft.

Dieser Widerspruch in Rodes Argumentation sollte aber nicht davon ablenken, daß es sich hier um eine sehr nützliche und engagierte Studie über ein aktuelles, wichtiges Thema handelt. Der Informationsreichtum und die gute Lesbarkeit dieser Studie bieten die Voraussetzungen dafür, daß ein weiter Leserkreis erreicht werden kann und daß kritische Denkimpulse bezüglich des vorherrschenden Südafrikabildes in der BRD vermittelt werden können.

\title{
Franz Ansprenger
}

\section{Die Befreiungspolitik der Organisation für}

\section{Afrikanische Einheit (OAU) 1963 bis 1975}

Studien zum Konflikt im südlichen Afrika. Entwicklung und Frieden.

Wissenschaftliche Reihe 8. München und Mainz 1975, S. 232.

Hier ist der - wie ich meine gelungene - Versuch unternommen worden, eine bisher nur in Umrissen bekannte und umstrittene Materie der afrikanischen Politik detailliert und übersichtlich darzustellen und in fairem Bemühen um das Verständnis der Mängel und Widersprüche der OAU-Politik zu kommentieren. Im Zentrum der Analyse steht die 13jährige Geschichte des Befreiungskomitees der OAU, des "African Liberation Comitee“ (ALC), das die Hilfeleistungen der afrikanischen Staaten an die kämpfenden Befreiungsbewegungen (vor allem im südlichen Afrika) koordinieren soll. In zunächst überraschender Weise - galt nicht der Beitrag der OAU zur Befreiung als marginal? - erfährt das OAU-Befreiungskomitee eine positive Würdigung; denn es hielt immerhin „die natürliche Tendenz jeder Exilpolitik zu immer weitergehender Zersplitterung in Grenzen“. Und „die OAU folgte in diesen Dingen immer den Beschlüssen der ALC, und die Vereinten Nationen folgten der OAU“ (S. 49). Vielleicht ist aber doch die andere Seite der OAU-Realität etwas zu kurz gekommen (obwohl die Kritik am ALC von links und rechts dargestellt wird), zu der etwa die Unfähigkeit gehört, mehr materielle Hilfe zu leisten oder aber die Entkolonialisierung Angolas konfliktreduzie- 
rend zu beeinflussen. Hätte die FRELIMO letztlich nicht auch ohne das ALC gesiegt?

Ansprenger dokumentiert die Prinzipien der OAU-Politik (gewaltsamer "nationaler" Kampf gegen Kolonialismus und Rassismus - aber nicht gegen "schlechte“ Herrschaft oder Diktaturen) sowie ihre einzelnen Etappen - von Nkrumahs Zeiten bis zum Manifest von Lusaka und der Dialog-Offerte Pretorias. Er reflektiert die Erfolgsbedingungen für die nationalen Befreiungskämpfe in den sechs umkämpften Territorien unter weißer Herrschaft. Aus dem Gestrüpp sich oftmals widersprechender Informationen über die Befreiungsbewegungen, über ihre Organisation und Erfolge, versucht Ansprenger in kritischer Distanz ein wahrheitsgemäßes Bild der jeweils realen Situation zu zeichnen. Obwohl manches Urteil im einzelnen nicht überzeugt oder (noch) spekulativ bleiben muß, so erfährt der Leser doch eine Fülle wichtiger Detailinformationen. In Kombination mit dem Bemühen um ein auch theoretisch ausgewiesenes Verständnis der OAU-Politik ist dieses Buch Ansprengers ein unverzichtbarer Bestandteil für das Begreifen afrikanischer Dekoloniations- und Befreiungspolitik - ihrer Prinzipien, ihrer Schwächen, ihrer Hintergründe.

Das Interessanteste an diesem Buch (das dem Anspruch noch am ehesten gerecht wird, ein praktischer Beitrag zur handlungsorientierten Friedens- und Konfliktforschung zu sein) scheinen mir jedoch die "Thesen zur Weiterführung der Diskussion“ (S. 159-168) zu sein. Sie bringen zum einen eine Begründung für die These, die OAU sei ihrem Anspruch der Koordinierung der Befreiungspolitik weitgehend gerecht geworden, zum anderen umreißen sie die unterschiedlichen Befreiungsstrategien für die portugiesischen Kolonien einerseits, für Rhodesien und Namibia andererseits. Für letztere wird eine Kombination gewaltfreier und gewalttätiger Aktionen empfohlen.

Schließlich diskutiert Ansprenger in seiner ihm eigenen Art der kalkulierten Provokation die "Kernfrage“: "Welches Modell gilt für die Republik Südafrika?" Unter dem Hinweis, daß bei einer "nationalen Befreiung“ der RSA "gleichzeitig eine komplizierte moderne Industriewirtschaft in Gang gehalten werden " müßte überrascht er den Leser mit der „ketzerischen“ These, daß dies z. B. die Kommunisten nach DDR-Modell zu tun in der Lage wären: „Der Verfasser hält eine ähnliche Zukunft in Südafrika für durchaus denkbar, zumal die Diktatur und ihre Polizei nur ausgewechselt, nicht neu geschaffen werden müßten (auch in diesem Punkt stimmt die Parallele zur DDR).“ (S. 164).

Die gewollt provokative Spekulation Ansprengers (die - was die inhaltliche Aussage betrifft - freilich wenig für sich hat) ist auf dem Hintergrund seiner Fundamentalkritik an der westlichen Südafrikapolitik zu bewerten: „Der Westen hat die Stunde versäumt, in der er im südlichen Afrika mit seinen gesellschaftlichen Wertbegriffen und seiner wirtschaftlichen Dynamik das Erbe des weißen Rassismus hätte antreten können“ (S. 165). Jetzt sieht er die „Kontur eines unheilvollen Bildes" immer deutlicher entstehen (S. 75): Der Westen - allein — gegen die Dritte Welt und die Kommunistische Weltbewegung. Was aus der Perspektive der UNO-Abstimmung zutreffen mag, hat in der konkreten Realität der afrikanischeuropäischen Kooperation keinen Platz. Wenn auch die These Ansprengers, daß der Westen bei der Entkolonisierung im südlichen Afrika völlig versagt habe, auf breiten Konsens stoßen wird, so darf bezweifelt werden, daß es zweckvoll und sinnvoll ist, mit dem Hinweis auf die „kommunistische Gefahr“ in Afrika (wo 
denn?) den Westen zur „Umkehr“ zu bewegen! Kann man übersehen, daß das oft als moskau-abhängig gescholtene Guinea-Bissao bereits EG-assoziert ist (wann folgen Mozambique und Angola?)? Ist der Westen in Afrika nicht viel zu präsent - auf Kosten afrikanischer Self-Reliance?

Dikussionswürdig ist schließlich auch die Grundannahme Anprengers, daß auch in der RSA den Afrikanern die "nationale Befreiung " gelingen wird (im Sinne der Majority rule!) - obwohl doch alle Anzeichen eher für eine Balkanisierung oder Teilung des Landes sprechen.

Gerade auch wegen seiner provokanten und versuchsweise zukunftantizipierenden Thesen - die sich bei Ansprenger auf der Grundlage großer Sachkenntnis aufbauen - ist dieses aktuelle Buch für alle, die besorgt die Entwicklung im südlichen Afrika verfolgen, von großem Nutzen und besonderem Reiz: man ist aufgefordert weiterzudenken.

\section{LeOnhard Harding}

\section{Afrikanische Politik im südlichen Afrika}

Studien zum Konflikt im südlichen Afrika. Entwicklung und Frieden. Wissenschaftliche Reihe 9.

München und Mainz 1975, 210 S.

In seinem zweiten Beitrag zu den „Studien“ analysiert Harding die Organisationen und strategischen Ziele der afrikanischen Akteure, die entweder als Kollaborateure oder als radikale Verweigerer der weißen Minderheit in der RSA gegenüberstehen. Dabei werden interessante Informationen über die "Strategie der taktischen Anpassung " seitens der Homeland-Führer Mantanzima, Mangope und Buthelezi gegeben sowie über die damit konfligierende "Strategie des Disengagements", die vor allem von der schwarzen Studentenorganisation SASO und der „Black Consciousness"-Bewegung unter den urbanisierten Weißen außerhalb der Bantustans vertreten wird. Dabei gelingt es Harding recht überzeugend nachzuweisen, daß die (zunächst als Ausgangshypothese postulierte, einzig mögliche) Strategie der Anpassung der Homeland-Leader an die Strukturen des von den Weißen erzwungenen "Separate development" das gew ünschte Ziel - den Systemwechsel — nicht erreichen könne, „aus politischen, vor allem aber aus wirtschaftlichen und noch stärker aus militärischen Gründen " (S. 75).

In der (beeindruckend dokumentierten) Black Consciousness-Bewegung in den Städten sieht Harding "Elemente einer prärevolutionären Situation“ verwirklicht, fügt aber hinzu, daß diese Strategie der Verweigerung (mit deutlichen Unterströmungen von Black Power und schwarzem Rassismus) „auf absehbare Zeit ohne klar definierte Erfolgschancen" sei (S. 98).

Auch die Nachbarstaaten - BLS-Länder, Malawi und Sambia - werden in die Betrachtung herrschaftsverändernder Prozesse und Perspektiven einbezogen, wobei der Autor z. B. bezüglich Sambias zu dem freilich (nicht gerade umwerfenden) Schluß gelangt: Im Interesse einer Konfliktlösungsstrategie ergäbe sich für die BRD als Ansatz einer „Interventionsmöglichkeit“, „Sambia bei seinem Bemühen um ein Disengagement vom Süden zu unterstützen, um auf diese Weise Pretorias Vordringen zum Norden zu stoppen und einen Machtwechsel in Rhodesien zu beschleunigen" (S. 132). Als wenn die RSA auf die Tomatenexporte nach Sambia angewiesen wäre! 